\title{
Efficacy and safety of switching from
} nevirapine immediate-release twice daily to nevirapine extended-release once daily in virologically suppressed HIV-infected patients: a retrospective cohort study in Taiwan

Chun-Yuan Lee ${ }^{1,2,3+}$, Hui-Min Chang ${ }^{4 \dagger}$, Calvin M Kunin ${ }^{5,6}$, Susan Shin-Jung Lee ${ }^{7,8}$, Yao-Shen Chen ${ }^{7,8,9}$ and Hung-Chin Tsai ${ }^{7,8^{*}}$

\begin{abstract}
Background: Whether the non-inferior efficacy and safety results of switching virologically suppressed HIV-1-infected patients from nevirapine immediate-release (NVP-IR) to NVP extended-release (NVP-XR) demonstrated in the TRANXITION study conducted in Europe and North America are also applicable to virologically suppressed HIV-infected Taiwanese patients remains unknown. We evaluated the comparative safety and efficacy of continuing NVP-IR versus switching to NVP-XR in virologically suppressed HIV-infected Taiwanese adults receiving combined antiretroviral therapy (CART) regimens.

Methods: We conducted a retrospective cohort study at Kaohsiung Veterans General Hospital from April 1, 2013, to March 31, 2015. Eighty-four virologically suppressed HIV-infected adults receiving NVP-IR cART were split into two groups: those continuing with NVP-IR $(n=49)$ and those being switched to NVP-XR $(n=35)$. Demographic characteristics, clinical variables, and laboratory findings were compared. Therapeutic drug monitoring of steady-state plasma NVP concentrations and genotype analysis of CYP2B6 516 were also performed in 22 participants. The primary endpoint was continued virological suppression at the end of the study. Secondary endpoints were time to loss of virological response and adverse events.

(Continued on next page)
\end{abstract}

\footnotetext{
* Correspondence: hctsai1011@yahoo.com.tw

${ }^{\dagger}$ Equal contributors

${ }^{7}$ Division of Infectious Diseases, Department of Medicine, Kaohsiung Veterans

General Hospital, Kaohsiung, Taiwan

${ }^{8}$ Faculty of Medicine, School of Medicine, National Yang-Ming University,

Taipei, Taiwan

Full list of author information is available at the end of the article
} 
(Continued from previous page)

Results: During a mean follow-up of 18.4 months, the NVP-XR group demonstrated similar success at maintaining virological response compared with the NVP-IR group ( $82.9 \%$ vs. $85.7 \%$; $P=0.72$ ). Cox regression analysis indicated that there were no significant differences between NVP regimens for time to loss of virological response (hazard ratio: 0.940; $P=0.754$ ). Furthermore, there were no significant differences in adverse events between these two groups. In the 22 participants, there was a non-significantly lower level of steady-state plasma NVP concentrations in the NVP-XR group than in NVP-IR recipients $(5145.0 \mathrm{ng} / \mathrm{mL}$ vs. $6775.0 \mathrm{ng} / \mathrm{mL}$; $P=0.267)$. The prevalence of CYP2B6 516 GT was $86.6 \%$, and there was no significant difference in the distribution of CYP2B6 516 between these two groups.

Conclusions: We found that switching from NVP-IR to NVP-XR appeared to have similar safety and efficacy compared with continuing NVP-IR among virologically suppressed, HIV-infected Taiwanese patients. Our finding of higher $C_{\text {trough }}$ levels in both groups compared with other studies conducted in Caucasian populations and the high prevalence of CYP2B6 516 GT requires further investigation in a larger Taiwanese cohort.

Keywords: Antiretroviral therapy, Human immunodeficiency virus, Nevirapine

\section{Background}

Nevirapine immediate-release (NVP-IR) has been used with a high level of efficacy and safety since its introduction in 1996 in the United States and 1998 in Europe as a potent non-nucleoside reverse transcriptase inhibitor (nNRTI) used in combined antiretroviral therapy (cART) [1-3]. However, efforts to simplify the regimens in terms of pill counts and frequency have continued to improve adherence to HAART $[4,5]$. Therefore, although the licensed dosage for NVP-IR is $200 \mathrm{mg}$ twice daily after a $200 \mathrm{mg}$ daily lead-in period, an off-label maintenance dose of $400 \mathrm{mg}(2 \times 200 \mathrm{mg})$ NVP-IR has been adopted to improve compliance [6]. Clinical trials comparing a single daily dose of $400 \mathrm{mg}$ NVP-IR versus $200 \mathrm{mg}$ twice daily demonstrated non-inferior efficacy for the new treatment [6-8]. To further simplify treatment regimens, a new extended-release (XR) formulation was developed $[9,10]$. In phase III clinical trials, $400 \mathrm{mg}$ NVP-XR once daily was found not inferior to $200 \mathrm{mg}$ NVP-IR twice daily in terms of virological suppression in treatmentnaïve patients (VERxVE study) [11] and in virologically suppressed patients who were switched from NVP-IR to NVP-XR (TRANxITION study) [12].

Nevirapine is pharmacologically characterized by a strong relationship between NVP trough plasma concentrations and virological response, $[13,14]$ and a target therapeutic trough concentration of $3.0 \mathrm{mg} / \mathrm{L}$ has been proposed as a minimum effective concentration [15] because the risk of virological failure increases 5-fold (relative risk 5.0, 95\% CI 1.8-13.7) with NVP $C_{\text {trough }} \leq 3 \mathrm{mg} / \mathrm{mL}$ compared with patients who had higher trough concentrations [13, 14].

However, in routine clinical practice, optimal plasma concentrations of NVP are reached in only $27.8 \%$ patients [16]. The significant inter-individual variability in plasma concentrations of nNRTI could be partially explained by differences in ethnicity, polymorphisms in enzymes, drug-drug interactions, and body weight [17-19]. Polymorphisms of $C Y P 2 B 6$ may influence the metabolism of NVP, with the variant CYP2B6 516 GT polymorphism resulting in reduced clearance of NVP compared with CYP2B6 516 GG [20]. In a 2NN pharmacokinetic (PK) substudy, NVP clearance was reduced by $19.4 \%$ in patients from Thailand and South Africa compared with Caucasian and Hispanic patients [20]. This difference has been related to CYP2B6 516 GT polymorphisms, resulting in a $15.3 \%$ reduced clearance of NVP compared with patients with CYP2B6 516 GG [20]. However, a recent study of 171 HIV-infected patients in northern Taiwan showed a predominance of homozygous 516 GG alleles (66.1\%), [21] which may lead to reduced NVP $C_{\text {trough }}$ compared with patients with CYP2B6 516 GT. Furthermore, a recent prospective study of $227 \mathrm{HIV}$-infected, treatment-naïve patients conducted in China recommends a higher target therapeutic $\mathrm{C}_{\text {trough }}(3.9 \mu \mathrm{g} / \mathrm{mL})$ of NVP for Chinese patients than the currently recommended level $(3.0 \mu \mathrm{g} / \mathrm{mL})$, which is predominately from the results of studies among Caucasian and African American patients [22].

In addition, previous PK studies of NVP have demonstrated a lower $C_{\text {trough }}$ with $400 \mathrm{mg}$ NVP-XR once daily compared with $200 \mathrm{mg}$ NVP-IR twice daily $[11,12,23]$. Therefore, the question remains whether the non-inferior efficacy and safety results of switching virologically suppressed HIV-1-infected patients from NVP-IR to NVP-XR demonstrated in the TRANxITION study conducted in Europe and North America [12] are also applicable to virologically suppressed HIVinfected patients in Taiwan.

The current study, conducted at a large teaching medical center in southern Taiwan, was designed to evaluate the efficacy and safety of switching virologically suppressed Taiwanese patients from $200 \mathrm{mg}$ NVP-IR twice daily to $400 \mathrm{mg}$ NVP-XR once daily. Therapeutic drug 
monitoring of plasma NVP concentrations and genotype analysis of CYP2B6 516 were also performed in some participants.

\section{Methods}

\section{Study design and patients}

This study is composed of two parts: a retrospective analysis of the efficacy and safety of switching virologically suppressed patients from $200 \mathrm{mg}$ NVP-IR twice daily to $400 \mathrm{mg}$ NVP-XR once daily, and a prospective analysis of the impact of CYP450 polymorphisms on plasma concentrations of nNRTI. In the first part of the study, we retrospectively examined data collected from April 1, 2013, to March 31, 2015, at Kaohsiung Veterans General Hospital (KVGH), a 1200-bed, general and tertiary care hospital located in southern Taiwan. Prior to April 2013, only NVP-IR was available at KVGH. Once NVP-XR became available at KVGH in April 2013, all patients receiving NVP-IR were queried by their physician regarding their willingness to switch to NVP-XR. Because HIV-infected patients regularly visit our infectious diseases department every 1-3 months, we retrospectively screened all HIV-infected patients who were receiving NVP-IR-containing cART during the period from April 1, 2013 to June 30, 2013. Inclusion criteria for virological suppression were patients who were receiving NVP-IR plus two nucleos(t)ide reverse-transcriptase inhibitors, for a preceding minimum of 18 weeks, with undetectable (<50 HIV-1 RNA copies/mL) HIV-1 viral load (VL) in the previous 1-4 months [12]. Exclusion criteria were age $<18$ years, pregnancy, or patients whose regimen included $400 \mathrm{mg}$ NVP-IR once daily/switching from NVP-IR to NVP-XR/change of the NRTI backbone of combination regimens between July 1, 2013, and March 31, 2015. Enrolled patients were further subdivided into those continuing NVP-IR twice daily and those switching to NVP-XR once daily.

For the second part of the study, we evaluated the impact of CYP450 polymorphisms on plasma concentrations of nNRTI by high-performance liquid chromatography (HPLC) in HIV-infected patients, beginning in January 1, 2014. Inclusion criteria were patients who were receiving the same nNRTI-containing cART for at least 14 days. We excluded patients who were also receiving medications other than antiretroviral agents, those who were aged $<20$ years, and those who did not adhere to cART. For patients who were eligible and agreed to participate, a steady-state plasma sample was obtained before administration of the next dose of nNRTI. Finally, we matched those patients enrolled in the prospective part of our study to those in the retrospective analysis.

The two parts of this study were approved by the KVGH ethics committee (VGHKS14-CT7-22 and
VGHKS14-CT2-13) and adhered to the principles of the Declaration of Helsinki. Informed consent was obtained from participants who underwent therapeutic drug monitoring of plasma nNRTI concentrations and genotype analysis of CYP450 polymorphisms.

\section{Data collection}

For each enrolled patient, the following information was extracted for analysis from computerized hospital records and chart reviews: demographic characteristics, risk factors for HIV infection, comorbidities, and cART regimen. Laboratory testing included hemograms, biochemical profiles, plasma viral load, and CD4+ T cell counts at baseline and at each follow-up visit. Viral load was determined using the Roche COBAS Amplicor assay prior to 2008 and the Roche COBAS TaqMan assay subsequently. Adverse events (AE) were also recorded. Liver function abnormalities were graded according to definitions of the Division of Acquired Immunodeficiency Syndrome (DAIDS).

\section{Definitions}

Virological suppression was defined as HIV RNA $\leq 50$ copies/mL. A virological blip was defined as an isolated detectable HIV RNA signal after a period of suppression, followed by a return to virological suppression [24]. Virological failure was defined as two consecutive HIV-1 RNA measurements $>50$ copies $/ \mathrm{mL}$ at least 2 weeks apart (also called a "double blip") [12]. Baseline data were defined as the most recent available test result within 3 months prior to entering the study. The date of entering the study was defined as April 1, 2013 for the NVP-IR group and as the date of switching for the NVP-XR group. The duration of patient follow-up was defined as the time from entering the study to treatment failure or to the last VL measurement within the observation period. The duration of continued virological suppression before entering the study was from the first VL measurement of $\leq 50$ copies $/ \mathrm{mL}$ to the date of entering the study.

\section{Study end points}

The primary end point was the proportion of patients with continued virological response with VL $<50$ HIV-1 RNA copies/mL at the end of the study. Patients were classified as treatment failures at the first occurrence of virological failure or a change in NVP regimen because of AEs or other reasons, death, or loss to follow-up (LTFU), [12] which was defined as no follow-up visits within 3 months prior to the end of the study. Secondary end points were time to loss of virological response (TLOVR) and AEs. 


\section{Therapeutic drug monitoring of steady-state plasma NVP concentrations}

A steady-state plasma sample was obtained from all participants before administration of the next dose of NVP. Blood samples were centrifuged immediately after collection, and plasma was removed and stored at $-20{ }^{\circ} \mathrm{C}$ until analysis. We used an HPLC technique with UV detection for analysis of NVP concentrations, as previously described [25]. For each calibration curve, six standards with concentrations ranging from 0.1 to $25 \mu \mathrm{g} / \mathrm{mL}$ were used. The lower limit of quantification of the assay was $0.1 \mu \mathrm{g} / \mathrm{mL}$. Intra- and interassay variabilities were $<5 \%$. Data were recorded and analyzed using HPLC 2D Chem Station software (Agilent Technologies, Santa Clara, CA, USA).

\section{Genotype analysis of CYP2B6 516}

Genomic DNA was isolated from peripheral blood using the PureLink Genomic DNA Mini Kit (Invitrogen, Carlsbad, CA, USA). Exon 4 of the CYP2B6 gene was amplified by polymerase chain reaction (PCR). The primers 5' CTTGACCTGCTGCTTCTTCC 3' and 5' TCCCTCTCCGTCTCCCTG 3' were used to amplify a 204-bp fragment. PCR products were digested with BsrI restriction enzyme (New England Biolabs, Inc., Beverley, MA, USA). Digestion products were then loaded on a $2 \%$ agarose gel and separated by gel electrophoresis [26, 27].

\section{Statistical analyses}

All statistical analyses were performed using IBM SPSS software, version 22.0 (IBM Corp., Armonk, NY, USA). Categorical variables were compared between the two groups using the $X^{2}$ or Fisher's exact tests, and continuous variables were compared using the independent $t$-test or Mann-Whitney $U$ test. All tests were two-tailed, and $P<0.05$ was considered significant. The TLOVR of these two groups was analyzed using a Kaplan-Meier survival curve, and the influence of the NVP regimen (NVP-IR twice daily vs. NVP-XR once daily) on the TLOVR was investigated using Cox regression that was adjusted for age, sex, history of AIDS, cART regimen, and duration of virological suppression.

\section{Results}

\section{Demographic characteristics and comorbidities of study participants}

We retrospectively screened the records of 170 patients who were receiving NVP-IR during the period April 1 to June 30, 2013. Ninety met the inclusion criteria for virological suppression. Overall, six of these patients were excluded because they were $<18$ years old $(n=1)$, receiving a regimen that contained $400 \mathrm{mg}$ NVP-IR once daily $(n=2)$, switching from NVP-IR to NVP-XR $(n=2)$, or had a change of the NRTI backbone $(n=1)$ between July 1, 2013, and March 31, 2015 (Fig. 1). Of the 84 patients enrolled in this study, 49 (59\%) continued a regimen of NVP-IR twice daily and 35 (41\%) were switched to an NVP-XR once daily regimen during the period from April 1 to June 30, 2013.

The baseline demographic characteristics and comorbidities of the two participant groups are shown in Table 1 . The median age was 41 years ( $\mathrm{SD} \pm 13$ years); 72 participants $(85.7 \%)$ were male. There were no statistically significant differences between the NVP-IR and NVP-XR groups with respect to age, sex, risk factors for HIV, comorbidities, duration of continued virological suppression, history of a virologic blip, baseline CD4+ counts, or patient follow-up. However, the two groups differed significantly in the NRTI backbone of combination regimens at baseline. Most patients in the NVP-IR group were treated with lamivudine and zidovudine (Combivir ${ }^{\circ}$ ) (NVP-IR vs. NVPXR: $55.1 \%$ vs. $0 \% ; P<0.001)$ whereas most patients in the NVP-XR group received abacavir and lamivudine (Kivexa) (NVP-XR vs. NVP-IR: $77.1 \%$ vs. $32.7 \%$; $P<0.001)$. Regarding the third drug of cART before entering the study, 24 of 35 patients (68.6\%) in the NVP-XR group received an off-label regimen with $400 \mathrm{mg}$ NVP-IR once daily, and all 49 patients in the NVP-IR group received $200 \mathrm{mg}$ NVP-IR twice daily.

\section{Outcomes of primary and secondary study end points}

Compared with those in the NVP-IR group, virologically suppressed HIV-infected participants in the NVP-XR group demonstrated more or less similar success at maintaining virological response during the mean follow-up time of 18.4 months (NVP-XR vs. NVP-IR: $82.9 \%$ vs. $85.7 \% ; P=0.72$ ) (Table 2 ). No patients in either group died during the observation period. In the NVP-XR group, one patient developed virological failure, which may have been as a result of poor compliance with cART, according to the chart review. Two patients were withdrawn from NVP-XR: one was suspected of having a hypersensitivity reaction to NVP-XR, and the other was owing to an insufficient supply of the drug. Three patients in the NVP-XR group were lost to follow-up for unknown reasons. In the NVP-IR group, one patient suffered from virological failure. Two patients were withdrawn from NVP-IR because they had NVP-induced hepatitis (one with DAIDS grade 2 and the other, grade 3). Four patients in the NVP-IR group were lost to follow-up. During the observation period, six patients (7.1\%) experienced isolated viral blips after entering the study (NVP-XR vs. NVP-IR: $11.4 \%$ vs. $4.1 \%$; $P=0.23)$. There were no differences in CD4+ count changes between the two groups (NVP-XR vs. NVP-IR: 23 cells $/ \mathrm{mm}^{3}$ vs. 76 cells $\left./ \mathrm{mm}^{3} ; P=0.32\right)$. 


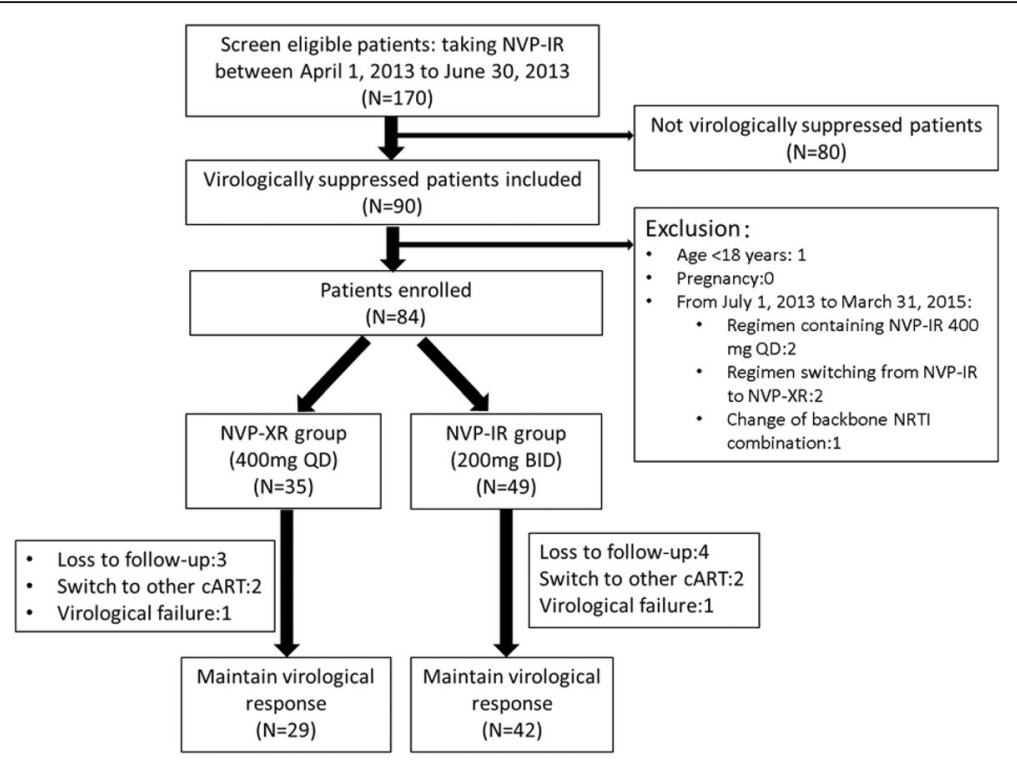

Fig. 1 Study design

The TLOVR for the two groups is shown in Fig. 2. There were no differences between these groups by log-rank test $(P=0.75)$. A Cox model adjusted for age, sex, history of AIDS, cART regimen, and duration of virological suppression demonstrated a nonsignificant difference between the NVP regimens with respect to hazard ratios (HR) for TLOVR (NVP-XR vs. NVP-IR: HR $0.940,95 \%$ CI $0.254-3.484$; $P=0.926)$ (Additional file 1).

\section{Adverse events among study participants}

There were no significant differences in AEs between the NVP-XR and NVP-IR groups (Table 3). Observed AEs included gastrointestinal complaints, skin rashes, and liver function abnormalities. One patient in the NVP-XR group found remnants of NVP-XR tablets in his stool. Three of 84 patients (3.6\%) discontinued their NVP regimen owing to AEs (Table 2). One patient taking NVP-XR experienced impaired liver function (DAIDS grade 1), low-grade fever, and skin rash 2 weeks after switching, suggesting a hypersensitivity reaction; two patients taking NVP-IR experienced NVP-induced hepatitis (DAIDS grade 2 and grade 3 ) that improved after switching to atazanavir. The majority of liver function abnormalities were mild (DAIDS grade 1). Higher grade liver function abnormalities (DAIDS grade $\geq 2$ ) were infrequent (NVP-XR vs. NVP-IR: $2.9 \%$ vs. $4.1 \% ; P=1.0)$. There was one case $(2.9 \%)$ of grade 4 aspartate transaminase/alanine transaminase levels in the NVP-XR group.
Therapeutic drug monitoring of steady-state plasma NVP concentration: Comparison between NVP-XR and NVP-IR groups

A total of 48 patients at KVGH were enrolled, to evaluate the impact of $C Y P 2 B 6516$ polymorphisms on plasma concentrations of NVP from January 1, 2014 to December 31, 2014. A total of 22 patients (22/48, 45.83\%, Additional file 2) were matched to patients enrolled in the retrospective part of the current study (22/84, 26.19\%), eight patients from the NVP-IR group and 14 from the NVP-XR group. There were no significant differences between these groups in age, sex, height, or weight (Additional file 3). Steady-state plasma NVP concentrations were lower in the NVP-XR group, but this difference did not reach statistical significance (NVP concentration (interquartile range): NVP-XR vs. NVP-IR, $5145.0 \mathrm{ng} / \mathrm{mL}(4070.0-5740.0 \mathrm{ng} / \mathrm{mL})$ vs. $6775.0 \mathrm{ng} / \mathrm{mL} \quad(4925.0-8380.0 \mathrm{ng} / \mathrm{mL}) ; \quad P=0.267)$ (Additional file 3).

\section{Genotype analysis of CYP2B6 516: Comparison between NVP-XR and NVP-IR groups}

Among the 22 patients participating in monitoring of steady-state plasma NVP concentrations, genotype analysis of CYP2B6 516 was conducted in 15 (15/22, 68.2\%) (Additional file 4); genotype analysis was not performed in the remaining seven patients owing to samples with insufficient residual volume. The prevalence of CYP2B6 516 GT was $86.6 \%(13 / 15)$. There was no significant difference in the distribution of CYP2B6 516 between these two groups (Additional file 3). 
Table 1 Demographic characteristics and co-morbidities for the 84 virologically suppressed, HIV-infected patients entered in this study

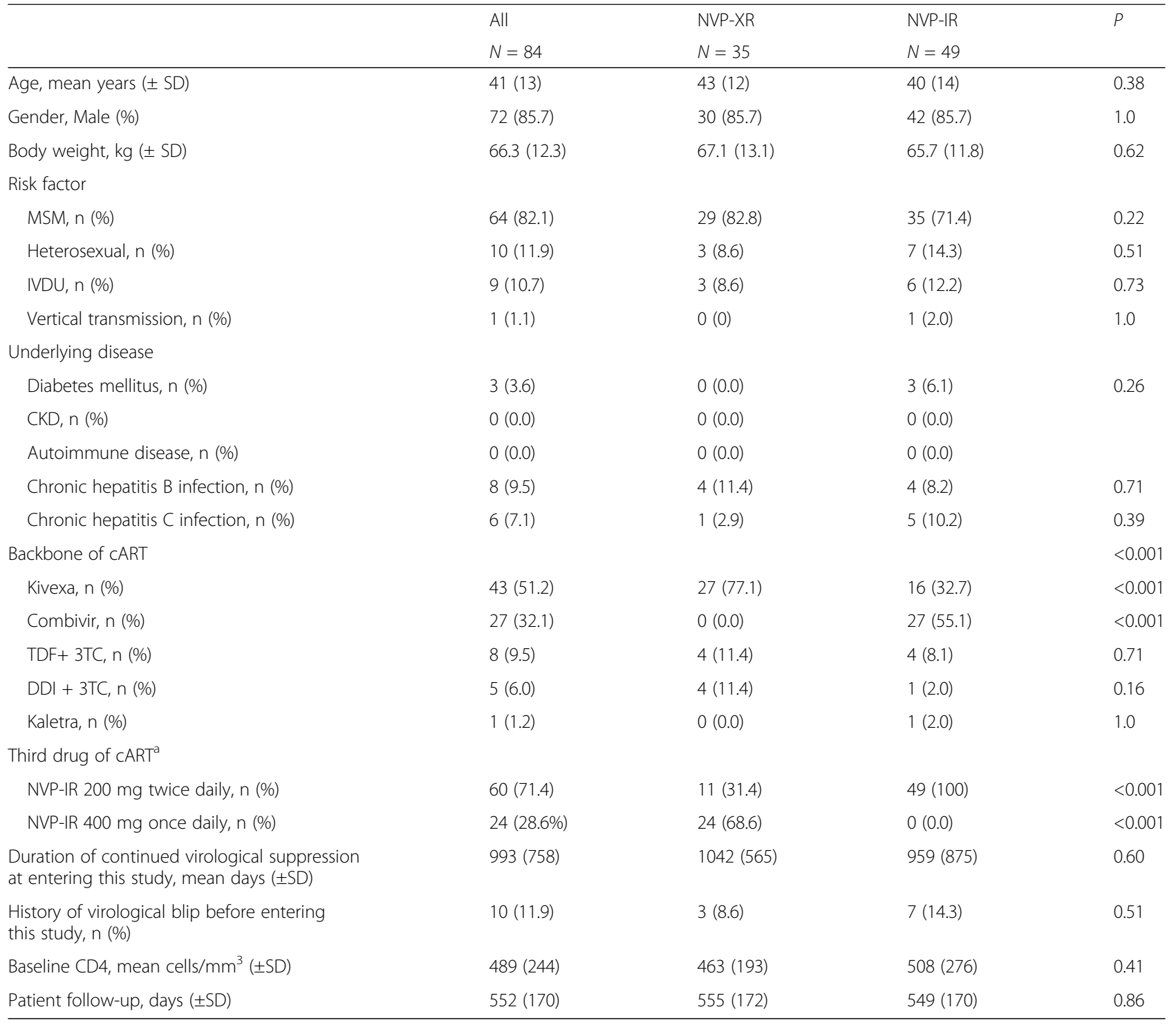

MSM men who have sex with men, CKD chronic kidney disease, DDI didanosine, IVDU intravenous drug user, NVP nevirapine, SD standard deviation, TDF tenofovir, 3TC lamivudine

${ }^{a}$ In the NVP-XR group, this means the nNRTIs prescribed prior to switching to NVP-XR-containing HAART

\section{Discussion}

To the best of our knowledge, this small retrospective cohort is the first to investigate the safety and efficacy of switching to NVP-XR from NVP-IR among virologically suppressed HIV-infected patients in Taiwan. Although the TRANxITION study demonstrated non-inferior efficacy and safety of switching virologically suppressed HIV-1-infected patients from NVP-IR to NVP-XR, the results were derived mainly from a Caucasian population. In this retrospective cohort study of virologically suppressed HIV-infected Taiwanese patients, we found that the NVP-XR group was similar to the NVP-IR group with respect to maintaining a virological response. The difference may not have been significant because the numbers of patients were relatively small and the study lacked the power to demonstrate a statistically significant difference. Despite methodological differences, the results of our comparative analyses of virological response indicated worse results compared with those of the TRANxITION study [12]. During NVP-IR therapy, $85.7 \%$ and $92.6 \%$ of patients in the current and TRANxITION study, respectively, were virologically suppressed. During NVP-XR therapy, 82.9 and $93.6 \%$ of patients in the current and TRANxITION studies, respectively, were virologically suppressed. The lower rate of virological response in our study may result from a longer follow-up period, as the mean follow-up times for the current and TRANxITION studies were 
Table 2 Outcomes for the 84 virologically suppressed HIV-infected patients entered in this study

\begin{tabular}{|c|c|c|c|c|}
\hline & All & NVP-XR & NVP-IR & $P$ \\
\hline & $N=84$ & $N=35$ & $N=49$ & \\
\hline Ability to maintain a virological response, $\mathrm{n}(\%)$ & $71(84.5)$ & $29(82.9)$ & $42(85.7)$ & 0.72 \\
\hline \multicolumn{5}{|l|}{ Reason for treatment failure } \\
\hline Death, n (\%) & $0(0.0)$ & $0(0.0)$ & $0(0.0)$ & \\
\hline $\begin{array}{l}\text { Switch to other CART due to adverse effect } \\
\text { or other cause, } n(\%)\end{array}$ & $4(4.8)$ & $2(5.7)^{a}$ & $2(4.1)$ & 1.00 \\
\hline Virological failure, n (\%) & $2(2.4)$ & $1(2.9)$ & $1(2.0)$ & 1.00 \\
\hline Loss to follow-up, n (\%) & $6(7.1)$ & $3(8.6)$ & $4(8.1)$ & 1.00 \\
\hline Virological blip, n (\%) & $6(7.1)$ & $4(11.4)$ & $2(4.1)$ & 0.23 \\
\hline $\begin{array}{l}\text { Change from baseline in CD4 T-cells, mean } \\
\text { cells } / \mathrm{mm}^{3}( \pm \mathrm{SD})\end{array}$ & $14(122)$ & $23(171)$ & $76(127)$ & 0.33 \\
\hline
\end{tabular}

CART combined antiretroviral therapy, NVP nevirapine, SD standard deviation

${ }^{a}$ One patient in the NVP-XR group discontinued NVP-XR because of insufficient supply of the drug

respectively 18.4 and 6 months. Furthermore, the current study was conducted outside a well-controlled trial setting. Therefore, the efficacy of NVP-IR and NVP-XR in maintaining virological response may be underestimated here owing to a relatively high proportion of LTFU treatment failures in both groups (NVP-IR vs. NVP XR: $50 \%(3 / 6)$ vs. $57 \%(4 / 7))$, which is inherent to retrospective studies.

Because previous studies have demonstrated a significant relationship between NVP trough plasma concentrations and virological response, $[13,28]$ we further investigated the NVP trough concentrations in both NVP-IR and NVP-XR groups. The median of trough plasma concentrations was $6775.0 \mathrm{ng} / \mathrm{mL}$ for NVP-IR and $5145.0 \mathrm{ng} / \mathrm{mL}$ for NVP-XR in our study (Additional file 3 ). The plasma concentrations of NVP in both groups are higher than the $\mathrm{C}_{\text {trough }}$ of $3.0 \mathrm{mg} / \mathrm{L}$ recommended in most guidelines [15] or $C_{\text {trough }}$ of $3.9 \mu \mathrm{g} / \mathrm{mL}$ for Chinese patients in a recent study [22]. The non-significant difference of NVP trough concentrations between these two groups may be owing to the small sample size in each group. This finding is compatible with previous studies, which demonstrated a lower $\mathrm{C}_{\text {trough }}$ with NVP-XR therapy compared with $200 \mathrm{mg}$ NVP-IR twice daily $[11,23]$.

We believe that there was no obvious selection bias between patients receiving NVP-XR and NVP-IR who also underwent therapeutic drug monitoring of steadystate plasma NVP concentration in the current study. Compliance, drug-drug interactions, and differences in pharmacokinetics are critical factors that influence the plasma concentrations of antiretroviral drugs [29]. Our patient population was assumed to be reasonably compliant because they had maintained a stable virological response to NVP-containing cART prior to entering the

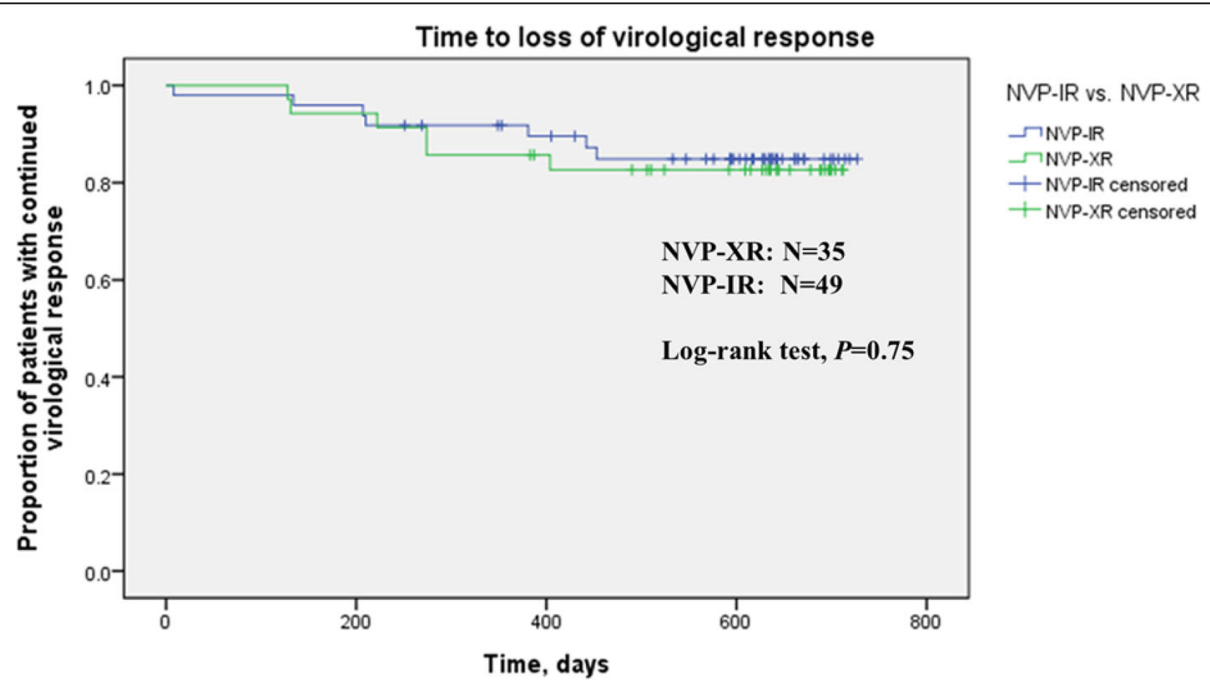

Fig. 2 Kaplan-Meier survival curves, time to loss of virological response in NVP-XR and NVP-IR groups. No significant difference was detected between the two treatment groups by log-rank test $(P=0.75)$ 
Table 3 Adverse effects and liver function abnormality tests of the 84 virologically suppressed HIV-infected patients entered in this study

\begin{tabular}{|c|c|c|c|c|c|c|c|c|c|}
\hline \multicolumn{10}{|c|}{ a Comparison of adverse effects between NVP-XR and NVP-IR } \\
\hline & All $N=84$ & \multicolumn{3}{|c|}{ NVP-XR N = 35} & \multicolumn{4}{|c|}{ NVP-IR N $=49$} & $P$ \\
\hline Skin rash, n (\%) & $1(1.2)$ & \multicolumn{3}{|l|}{$1(2.9)$} & \multicolumn{4}{|l|}{$0(0.0)$} & 0.42 \\
\hline Gastrointestinal disorders, n (\%) & $2(2.4)$ & \multicolumn{3}{|l|}{$1(2.9)$} & \multicolumn{4}{|l|}{$1(2.0)$} & 1.0 \\
\hline Tablet remnants in stools, $\mathrm{n}(\%)$ & $1(2.4)$ & \multicolumn{3}{|l|}{$1(2.9)$} & \multicolumn{4}{|l|}{$0(0.0)$} & 0.42 \\
\hline \multicolumn{10}{|c|}{ b Comparison of liver function abnormalities between NVP-XR and NVP-IR } \\
\hline & \multicolumn{4}{|c|}{ NVP-XR 400 mg qd $(N=35)$} & \multicolumn{4}{|c|}{ NVP-IR 200 mg bid $(N=49)$} & $P$ \\
\hline \multirow[t]{4}{*}{ AST elevation, $\mathrm{n}(\%)$} & \multicolumn{4}{|l|}{$6(17.1)$} & \multicolumn{4}{|l|}{$12(24.5)$} & 0.42 \\
\hline & Grade I & Grade II & Grade III & Grade IV & Grade I & Grade II & Grade III & Grade IV & \\
\hline & n (\%) & n (\%) & n (\%) & n (\%) & n (\%) & n (\%) & n (\%) & n (\%) & \\
\hline & $5(14.3)$ & $0(0)$ & $0(0)$ & $1(2.9)$ & $10(22.4)$ & $1(2.0)$ & $1(2.0)$ & $0(0)$ & 0.28 \\
\hline \multirow[t]{4}{*}{ ALT elevation, n (\%) } & $9(25.7)$ & & & & $16(32.7)$ & & & & 0.49 \\
\hline & Grade I & Grade II & Grade III & Grade IV & Grade I & Grade II & Grade III & Grade IV & \\
\hline & n (\%) & n (\%) & n (\%) & n (\%) & n (\%) & n (\%) & n (\%) & n (\%) & \\
\hline & $8(22.9)$ & $0(0)$ & $0(0)$ & $1(2.9)$ & $13(28.6)$ & $2(4.1)$ & $1(2.0)$ & $0(0)$ & \\
\hline \multirow[t]{4}{*}{ Bilirubin, n (\%) } & $1(2.9)$ & & & & $0(0)$ & & & & 0.42 \\
\hline & Grade I & Grade II & Grade III & Grade IV & Grade I & Grade II & Grade III & Grade IV & \\
\hline & n (\%) & n (\%) & n (\%) & n (\%) & n (\%) & n (\%) & n (\%) & n (\%) & \\
\hline & $1(2.9)$ & $0(0)$ & $0(0)$ & $0(0)$ & $0(0)$ & $0(0)$ & $0(0)$ & $0(0)$ & \\
\hline
\end{tabular}

AST aspartate aminotransferase, $A L T$ alanine aminotransferase

(a) Comparison of adverse effects between NVP-XR $(n=35)$ and NVP-IR $(n=49)$. (b) Comparison of liver function abnormalities between NVP-XR $(n=35)$ and NVP-IR $(n=49)$

study, and all participants remained virologically suppressed for at least 1-4 months (duration of continued virological suppression at entering this study, days $( \pm$ SD): NVP-XR vs. NVP-IR, 1042 (565) vs. 959 (875); $P=0.60$ ). Additionally, these patients only received antiretroviral agents and did not take any other prescribed medicine concomitantly. Finally, genotype analysis of CYP2B6 516 in 15 patients also revealed no significant difference in CYP2B6 516 polymorphisms between these two groups.

Surprisingly, however, our study demonstrated higher $\mathrm{C}_{\text {trough }}$ levels in both groups compared with other studies conducted in the Netherlands, [30] Germany, [31] and Canada [32]. This difference may result from a higher prevalence of CYP2B6 516 GT in our study population (86.6\%) compared with other reports of Han Chinese, [33] Caucasians, [29] and Taiwanese in northern Taiwan [21]. Our finding of a high prevalence of CYP2B6 $516 \mathrm{GT}$ in the current study requires further verification in a larger Taiwanese population.

Maximal and durable suppression of plasma HIV viral load has now been established as the primary aim of antiretroviral treatment for both treatment-naïve and treatment-experienced patients [24]. However, viral replication is still not fully controlled in all patients at all times, and transient viremia, often between 50 and 400 copies $/ \mathrm{mL}$ (a virological blip) has been frequently reported [34].
However, the impact of HIV RNA blips on virological failure remains controversial $[35,36]$. In the ACTG 343 and Merck 035 trials, treatment-experienced patients who had virological blips did not have an increased risk of virological failure compared with patients with continuing virological suppression [35]. However, the Swiss HIV Cohort and Frankfurt HIV Clinic Cohort studies showed a slightly increased risk of virological failure for patients with one or more virological blips compared with viral suppression (HR 2.01, 95\% CI 1.51-2.91; $P<0.0001$ ) [36]. In our cohort study, six of 84 patients (7.1\%) experienced a virologic blip during the 18.4-month follow-up. In Cox regressions adjusted for age, sex, history of AIDS, cART regimen, and history of virological suppression, the HR of viral blips was $1.023(P=1.00)$ for the NVP-XR group compared with the NVP-IR group. The relationship between viral blip and virological failure in Taiwanese patients requires further investigation in a larger population.

The current study has several limitations and biases inherent to retrospective cohort studies, the first being selection bias. Second, there were significant differences in cART backbone drugs between the NVP-XR and NVP-IR groups. However, the CNA30024 study comparing the durability of viral effect and the safety profile of triple therapy with either abacavir or zidovudine, combined with lamivudine and efavirenz, demonstrated similar virological suppression (HIV RNA $\leq 50$ copies $/ \mathrm{mL}$ ) in 
treatment-naïve patients at week 48 (abacavir group vs. zidovudine group: $70 \%$ vs. 69\%) [37]. Third, it is difficult to determine patient compliance with medications from retrospective chart reviews. This is important because adherence to treatment is closely related to sustained viral suppression [38]. As mentioned above, we believe that our patient population was reasonably compliant because only patients with virological suppression for at least 1-4 months were included. Finally, the frequency of adverse reactions to NVP in both groups may be underestimated. Although the frequency of moderate to severe liver function abnormalities is similar to that of the TRANxITION study, it is likely that we missed some low-grade skin rashes and gastrointestinal disorders that did not require modification of the antiretroviral regimen.

\section{Conclusions}

In this retrospective study, we found that switching from cART regimens with twice-daily doses of $200 \mathrm{mg}$ NVP-IR to a once-daily dose of $400 \mathrm{mg}$ NVP-XR appeared nearly as safe and effective as continuing NVP-IR in virologically suppressed HIV-infected Taiwanese patients. Our findings of higher $\mathrm{C}_{\text {trough }}$ levels in both groups compared with other studies conducted among Caucasian populations may be due to the high prevalence of CYP2B6 516 intermediate/ low metabolizers in the present study.

\section{Additional files}

Additional file 1: Multivariate analysis of treatment failure in 84 virologically suppressed HIV-infected patients. In Cox regression, the hazard ratios for TLOVR between NVP regimen (NVP-XR Vs. NVP-IR) was $0.940(95 \% \mathrm{Cl}$, $0.254-3.484 ; P=0.926)$. (DOCX $14 \mathrm{~kb})$

Additional file 2: Comparison of demographic characteristics, steady-state plasma concentration of NVP, and genotype analysis of CYP2B6 516 in 48 HIV-infected patients enrolled in KVGH from January 1, 2014 to December 31, 2014, by patients enrolled or not enrolled in the retrospective part of the current study. The 22 patients enrolled in the retrospective analysis of the current study were older (37 vs. $27.5, P<0.001$ ) and heavier (74.5 kg vs. $64.8 \mathrm{~kg}, P=0.021$ ) than the 26 patients not enrolled in the retrospective analysis of the current study. There were no significant differences between these two groups in sex, height, the steady-state plasma NVP concentration, and genotype analysis of CYP2B6 516. (DOCX $15 \mathrm{~kb}$ )

Additional file 3: Demographic characteristics, steady-state plasma concentration of NVP, and genotype analysis of CYP2B6 516 of 22 virologically suppressed, HIV-infected patients enrolled in this study, by NVP regimens. There were no significant differences between NVP-XR and NPV-IR in age, sex, height, weight, steady-state plasma NVP concentrations, and genotype analysis of CYP2B6 516. (DOCX $15 \mathrm{~kb}$ )

Additional file 4: $P C R$-restriction fragment length polymorphism analysis of CYP2B6 516. Three cases (A, B, C) participating in genotype analysis of CYP2B6 516 in the current study are shown. Lane M represents a size marker. After digestion with Bsil restriction enzyme, wild-type GG (case B) is visible as one band of 152 base pair. Heterozygous GT (case C) is visible as two bands (152 base pair and 204 base pair); homozygous mutant TT (case A) is visible as one band of 204 base pair. (PPTX $122 \mathrm{~kb}$ )

\section{Abbreviations}

AE: Adverse events; CART: Combined antiretroviral therapy; DAIDS: Division of Acquired Immunodeficiency Syndrome; HAART: Highly active antiretroviral therapy; HPLC: High-performance liquid chromatography; HR: Hazard ratio; KVGH: Kaohsiung Veterans General Hospital; LTFU: lost to follow-up; NRTI: Nucleoside reverse transcriptase inhibitor; NVP-IR: Nevirapine immediaterelease; NVP-XR: Nevirapine extended-release; PCR: Polymerase chain reaction; PK: Pharmacokinetic; TLOVR: Time to loss of virological response; VL: Viral load

\section{Acknowledgements}

Not applicable.

\section{Funding}

This study was funded by Veterans General Hospitals and University System of Taiwan Joint Research Programme grant.

\section{Availability of data and materials}

The datasets used and/or analyzed during the current study are available from the corresponding author on reasonable request.

\section{Authors' contributions}

$\mathrm{C}-\mathrm{YL}$ and $\mathrm{H}-\mathrm{MC}$ conceived and designed the study, and drafted the manuscript. CMK, S S-JL, and Y-SC analyzed and interpreted the data. H-CT analyzed and interpreted the data, and critically revised the manuscript. All authors read and approved the final manuscript.

\section{Competing interests}

The authors declare that they have no competing interests.

\section{Consent for publication}

Not applicable.

\section{Ethics approval and consent to participate}

This study was approved by the KVGH ethics committee (VGHKS14-CT7-22 and VGHKS14-CT2-13) and adhered to the principles of the Declaration of Helsinki. Informed consent was obtained from participants who received therapeutic drug monitoring of plasma nNRTI concentrations and genotype analysis of CYP450 polymorphisms.

\section{Publisher's note}

Springer Nature remains neutral with regard to jurisdictional claims in published maps and Institutional affiliations.

\section{Author details}

'Division of Infectious Diseases, Department of Internal Medicine, Kaohsiung Medical University, Kaohsiung Medical University Hospital, Kaohsiung, Taiwan. ${ }^{2}$ Center for Infectious Disease and Cancer Research (CICAR), Kaohsiung Medical University, Kaohsiung, Taiwan. ${ }^{3}$ Graduate Institute of Medicine, Kaohsiung Medical University, Kaohsiung, Taiwan. ${ }^{4}$ Department of Pharmacy, Kaohsiung Veterans General Hospital, Kaohsiung, Taiwan. ${ }^{5}$ Department of Internal Medicine, Ohio State University, Columbus, OH, USA. ${ }^{6}$ Department of Internal Medicine, University of Arizona, Tucson, AZ, USA. ${ }^{7}$ Division of Infectious Diseases, Department of Medicine, Kaohsiung Veterans General Hospital, Kaohsiung, Taiwan. ${ }^{8}$ Faculty of Medicine, School of Medicine, National Yang-Ming University, Taipei, Taiwan. ${ }^{9}$ Graduate Institute of Science Education and Environmental Education, National Kaohsiung Normal University, Kaohsiung, Taiwan.

Received: 5 September 2016 Accepted: 30 March 2017 Published online: 11 April 2017

\section{References}

1. Lockman S, Hughes M, Sawe F, Zheng Y, Mclntyre J, Chipato T, et al. Nevirapine- versus lopinavir/ritonavir-based initial therapy for HIV-1 infection among women in Africa: a randomized trial. PLoS Med. 2012;9:e1001236.

2. Soriano V, Arasteh K, Migrone H, Lutz T, Opravil M, Andrade-Villanueva J, et al. Nevirapine versus atazanavir/ritonavir, each combined with tenofovir disoproxil fumarate/emtricitabine, in antiretroviral-naive HIV-1 patients: the ARTEN trial. AntivirTher. 2011;16:339-48.

3. Waters L, John L, Nelson M. Non-nucleoside reverse transcriptase inhibitors: a review. Int J Clin Pract. 2007;61:105-18. 
4. Stone VE, Jordan J, Tolson J, Miller R, Pilon T. Perspectives on adherence and simplicity for HIV-infected patients on antiretroviral therapy: self-repor of the relative importance of multiple attributes of highly active antiretroviral therapy (HAART) regimens in predicting adherence. J Acquir Immune Defic Syndr. 2004;36:808-16.

5. Gallant JE, DeJesus E, Arribas JR, Pozniak AL, Gazzard B, Campo RE, et al. Tenofovir DF, emtricitabine, and efavirenz vs. zidovudine, lamivudine, and efavirenz for HIV. N Engl J Med. 2006;354:251-60.

6. Calmy A, Vallier N, Nguyen A, Lange JM, Battegay M, de Wolf F, et al. Safety and efficacy of once-daily nevirapine dosing: a multicohort study. Antivir Ther. 2009;14:931-8

7. van Leth F, Phanuphak P, Ruxrungtham K, Baraldi E, Miller S, Gazzard B, et al. Comparison of first-line antiretroviral therapy with regimens including nevirapine, efavirenz, or both drugs, plus stavudine and lamivudine: a randomised open-label trial, the 2NN study. Lancet. 2004; 363:1253-63.

8. Podzamczer D, Olmo M, Sanz J, Boix V, Negredo E, Knobel H, et al. Safety of switching Nevirapine twice daily to Nevirapine once daily in Virologically suppressed patients. J Acquir Immune Defic Syndr. 2009;50:390-6.

9. Macha S, Yong CL, Darrington T, Davis MS, MacGregor TR, Castles M, et al. In vitro-in vivo correlation for nevirapine extended release tablets. Biopharm Drug Dispos. 2009;30:542-50.

10. Quinson A, Arasteh K, Plettenberg A, Bogner J, Boue F, Livrozet JM, et al Steady state evaluation of two extended release (XR) nevirapine (NVP) tablets 400 mg QD compared with immediate release (IR) NVP tablets 200 mg BID in HIV-1 infected patients; 49th Interscience conference of antimicrobial agents and chemotherapy, 2009

11. Gathe J, Andrade-Villanueva J, Santiago S, Horban A, Nelson M, Cahn P, et al. Efficacy and safety of nevirapine extended-release once daily versus nevirapine immediate-release twice-daily in treatment-naive HIV-1-infected patients. Antivir Ther. 2011;16:759-69.

12. Arasteh $\mathrm{K}$, Ward D, Plettenberg A, Livrozet JM, Orkin C, Cordes C, et al. Twenty-four-week efficacy and safety of switching virologically suppressed HIV-1-infected patients from nevirapine immediate release $200 \mathrm{mg}$ twice daily to nevirapine extended release $400 \mathrm{mg}$ once daily (TRANxITION). HIV Med. 2012;13:236-44.

13. de Vries-Sluijs TE, Dieleman JP, Arts D, Huitema AD, Beijnen JH, Schutten M, et al. Low nevirapine plasma concentrations predict virological failure in an unselected HIV-1-infected population. Clin Pharmacokinet. 2003:42:599-605.

14. Veldkamp Al, Weverling GJ, Lange JM, Montaner JS, Reiss P, Cooper DA, et al. High exposure to nevirapine in plasma is associated with an improved virological response in HIV-1-infected individuals. AIDS. 2001;15:1089-95.

15. Higgins N, Tseng A, Sheehan NL, la Porte CJ. Antiretroviral therapeutic drug monitoring in Canada: current status and recommendations for clinical practice. Can J Hosp Pharm. 2009;62:500-9.

16. Moltó J, Blanco A, Miranda C, Miranda J, Puig J, Valle M, et al. Variability in non-nucleoside reverse transcriptase and protease inhibitors concentrations among HIV-infected adults in routine clinical practice. Br J Clin Pharmacol. 2007;63:715-21.

17. Chou M, Bertrand J, Segeral O, Verstuyft C, Borand L, Comets E, et al. Population pharmacokinetic-pharmacogenetic study of nevirapine in HIVinfected Cambodian patients. Antimicrob Agents Chemother. 2010;54:4432-9.

18. Stohr W, Back D, Dunn D, Sabin C, Winston A, Gilson R, et al. Factors influencing efavirenz and nevirapine plasma concentration: effect of ethnicity, weight and co-medication. Antivir Ther. 2008;13:675-85.

19. Schipani A, Wyen $C$, Mahungu $T$, Hendra H, Egan D, Siccardi M, et al. Integration of population pharmacokinetics and pharmacogenetics: an aid to optimal nevirapine dose selection in HIV-infected individuals. J Antimicrob Chemother. 2011;66:1332-9.

20. Lehr T, Yuan J, Hall D, Zimdahl-Gelling H, Schaefer HG, Staab A, et al. Integration of absorption, distribution, metabolism, and elimination genotyping data into a population pharmacokinetic analysis of nevirapine. Pharmacogenet Genomics. 2011;21:721-30.

21. Lee K-Y, Lin S-W, Sun H-Y, Kuo CH, Tsai MS, Wu BR, et al. Therapeutic drug monitoring and pharmacogenetic study of HIV-infected ethnic Chinese receiving efavirenz-containing antiretroviral therapy with or without rifampicin-based anti-tuberculous therapy. PLoS One. 2014;9:e88497.

22. Wang J, Kou H, Fu Q, Han Y, Qiu Z, Zuo L, et al. Nevirapine plasma concentrations are associated with virologic response and hepatotoxicity in Chinese patients with HIV infection. PLoS One. 2011;6:e26739.
23. Lepik KJ, Yip B, McGovern RA, Ding E, Nohpal A, Watson BE, et al. Postmarketing experience with nevirapine extended release (XR) tablets: effectiveness and tolerability in a population-based cohort in British Columbia, Canada. Antivir Ther. 2015;20:721-730.

24. Department of Health and Human Services. Guidelines for the use of antiretroviral agents in HIV-1-infected adults and adolescents; 2017. p. 1-288.

25. Rezk NL, Tidwell RR, Kashuba AD. Simultaneous determination of six HIV nucleoside analogue reverse transcriptase inhibitors and nevirapine by liquid chromatography with ultraviolet absorbance detection. J Chromatogr B Analyt Technol Biomed Life Sci. 2003;791:137-47.

26. Ariyoshi N, Miyazaki M, Toide K, Yi S, Kamataki T. A single nucleotide polymorphism of CYP2B6 found in Japanese enhances catalytic activity by autoactivation. Biochem Biophys Res Commun. 2001;281:1256-60.

27. Ramachandran G, Kumar AH, Rajasekaran S, Kumar P, Ramesh K, Anitha S, et al. CYP2B6 G516T polymorphism but not rifampin coadministration influences steady-state pharmacokinetics of efavirenz in human immunodeficiency virus-infected patients in South India. Antimicrob Agents Chemother. 2009;53:863-8

28. Duong M, Buisson M, Peytavin G, Kohli E, Piroth L, Martha B, et al. Low trough plasma concentrations of nevirapine associated with virologic rebounds in HIV-infected patients who switched from protease inhibitors. Ann Pharmacother. 2005:39:603-9.

29. Russo G, Paganotti GM, Soeria-Atmadja S, Haverkamp M, Ramogola-Masire $D$, Vullo $V$, et al. Pharmacogenetics of non-nucleoside reverse transcriptase inhibitors (NNRTIs) in resource-limited settings: influence on antiretroviral therapy response and concomitant anti-tubercular, antimalarial and contraceptive treatments. Infect Genet Evol. 2016;37:192-207.

30. van Heeswijk RP, Veldkamp Al, Mulder JW, Meenhorst PL, Wit FW, Lange JM, et al. The steady-state pharmacokinetics of nevirapine during once daily and twice daily dosing in HIV-1-infected individuals. AIDS. 2000;14:F77-82.

31. Von Hentig N, Carlebach A, Gute P, Knecht G, Klauke S, Rohrbacher M, et al. A comparison of the steady-state pharmacokinetics of nevirapine in men, nonpregnant women and women in late pregnancy. Br J Clin Pharmacol. 2006;62:552-9.

32. Lepik KJ, Yip B, McGovern RA, Ding E, Nohpal A, Watson BE, et al. Postmarketing experience with nevirapine extended release $(X R)$ tablets: effectiveness and tolerability in a population-based cohort in British Columbia, Canada. Antivir Ther. 2015;20:721-30.

33. Guan S, Huang M, Chan E, Chen X, Duan W, Zhou SF. Genetic polymorphisms of cytochrome P450 2B6 gene in Han Chinese. Eur J Pharm Sci. 2006;29:14-21.

34. Pozniak A, Gupta RK, Pillay D, Arribas J, Hill A. Causes and consequences of incomplete HIV RNA suppression in clinical trials. HIV Clin Trials. 2009;10: 289-98.

35. Havlir DV, Bassett R, Levitan D, Gilbert P, Tebas P, Collier AC, et al. Prevalence and predictive value of intermittent viremia with combination hiv therapy. JAMA. 2001;286:171-9.

36. Greub G, Cozzi-Lepri A, Ledergerber B, Staszewski S, Perrin L, Miller V, et al. Intermittent and sustained low-level HIV viral rebound in patients receiving potent antiretroviral therapy. AIDS. 2002;16:1967-9.

37. DeJesus E, Herrera G, Teofilo E, Gerstoft J, Buendia CB, Brand JD, et al. Abacavir versus zidovudine combined with lamivudine and efavirenz, for the treatment of antiretroviral-naive HIV-infected adults. Clin Infect Dis. 2004;39:1038-46.

38 Oette M, Kroidl A, Gobels K, Stabbert A, Menge M, Sagir A, et al. Predictors of short-term success of antiretroviral therapy in HIV infection. J Antimicrob Chemother. 2006:58:147-53. 